\title{
Optimal Regularity Properties of the Generalized Sobolev Spaces
}

\author{
G. E. Karadzhov ${ }^{1,2}$ and Qaisar Mehmood ${ }^{2}$ \\ ${ }^{1}$ Institute of Mathematics and Informatics, Bulgarian Academy of Sciences, 1113 Sofia, Bulgaria \\ ${ }^{2}$ Abdus Salam School of Mathematical Sciences, GC University, Lahore 68-B, Pakistan
}

Correspondence should be addressed to G. E. Karadzhov; geremika@yahoo.com

Received 21 May 2013; Accepted 16 September 2013

Academic Editor: Josip E. Pečarić

Copyright ( 2013 G. E. Karadzhov and Q. Mehmood. This is an open access article distributed under the Creative Commons Attribution License, which permits unrestricted use, distribution, and reproduction in any medium, provided the original work is properly cited.

We prove optimal embeddings of the generalized Sobolev spaces $W^{k} E$, where $E$ is a rearrangement invariant function space, into the generalized Hölder-Zygmund space $\mathscr{C} H$ generated by a function space $H$.

\section{Introduction}

The classical Sobolev space $W_{p}^{k}, 1 \leq p<\infty$, consists of all locally integrable functions $f$, defined on $\mathbf{R}^{n}, n \geq 1$, with the Lebesgue measure, such that the following norm is finite: $\|f\|_{W_{p}^{k}}=\sum_{|\alpha| \leq k}\left\|D^{\alpha} f\right\|_{p}$, where $\|f\|_{p}$ stands for the $L^{p}$-norm. In investigating the regularity of the function $f \in W_{p}^{k}$, we may assume, without any loss of generality, that $f \in L^{1}(\Omega)$, $\Omega$ is a domain in $\mathbf{R}^{n}$, and $f$ is zero outside $\Omega$. For simplicity we suppose that the Lebesgue measure of $\Omega$ equals one and that the origin lies in $\Omega$. It is well known that in the supercritical case $k>n / p$,

$$
W_{p}^{k} \hookrightarrow \mathscr{C}^{k-n / p}, \quad k>\frac{n}{p},
$$

where $\mathscr{C}^{\gamma}, \gamma>0$, is the Hölder-Zygmund space (see [1]). In the critical case $k=n / p$ the function $f \in W_{p}^{k}$ may not be even continuous. The result (1) is not optimal. We prove that the optimal one is obtained if in (1) $L^{p}$ is replaced by the Marcinkiewicz space $L^{p, \infty}$. In this paper we prove similar optimal results, when $L^{p, \infty}$ is replaced by a more general rearrangement invariant space $E$. The Sobolev space $W^{k} E$ consists of all $f \in W_{1}^{k}$ with a finite quasinorm $\|f\|_{W^{k} E}=$ $\sum_{|\alpha| \leq k}\left\|D^{\alpha} f\right\|_{E}$. More precisely, we consider quasinormed rearrangement invariant spaces $E$, consisting of functions $f \in L^{1}(\Omega)$, such that the quasinorm $\|f\|_{E} \approx \rho_{E}\left(f^{*}\right)<\infty$, where $\rho_{E}$ is a monotone quasinorm, defined on $M^{+}$with values in $[0, \infty]$ and $M^{+}$is the cone of all locally integrable functions $g \geq 0$ on $(0,1)$ with the Lebesgue measure. Monotonicity means that $g_{1} \leq g_{2}$ implies $\rho_{E}\left(g_{1}\right) \leq$ $\rho_{E}\left(g_{2}\right)$. We suppose that $L^{\infty}(\Omega) \hookrightarrow E \hookrightarrow L^{1}(\Omega)$, which means continuous embeddings. Here $f^{*}$ is the decreasing rearrangement of $f$, given by $f^{*}(t)=\inf \left\{\lambda>0: \mu_{f}(\lambda) \leq\right.$ $t\}, t>0$, and $\mu_{f}$ is the distribution function of $f$, defined by $\mu_{f}(\lambda)=\left|\left\{x \in \mathbf{R}^{n}:|f(x)|>\lambda\right\}\right|_{n},|\cdot|_{n}$ denoting Lebesgue $n$-measure. Note that $f^{*}(t)=0$ for $t>1$. Finally, $f^{* *}(t):=$ $(1 / t) \int_{0}^{t} f^{*}(s) d s$.

Let $\alpha_{E}, \beta_{E}$ be the Boyd indices of $E$. For example, if $E=$ $L^{p}$, then $\alpha_{E}=\beta_{E}=1 / p$ and the condition $k / n \geq 1 / p$ means $k / n \geq \alpha_{E}>0$. Note that for $k>n$ this is always satisfied. For these reasons we suppose that for the general $E, 0<\alpha_{E}=\beta_{E} \leq 1$ and the case $\min (k, n) / n>\alpha_{E}$ is called super-critical, while the case $\min (k, n) / n=\alpha_{E}$-critical. In the super-critical case the function $f \in W^{k} E$ is always continuous, while the spaces in the critical case $\alpha_{E}=k / n, k<$ $n$, can be divided into two subclasses: in the first subclass the functions $f \in W^{k} E$ may not be continuous-then the target space is rearrangement invariant, while these functions in the second subclass are continuous and the target space is the generalized Hölder-Zygmund space $\mathscr{C H}$ (see Definition 1). The separating space for these two subclasses is given by the Lorentz space $L^{n / k, 1}, k<n$. If $k \geq n$; then $W^{k} E$ consists of continuous functions (see the classical result of Stein [2]). 
The main goal of this paper is to prove optimal embeddings of the Sobolev space $W^{k} E$ into the generalized HölderZygmund space $\mathscr{C H}$. First we prove that this embedding for $k \leq n$ is equivalent to the continuity of the operator $R_{k} g(t)=\int_{0}^{t} u^{k / n-1} g(u) d u$. The case $k>n$ is reduced to the continuity of $R_{n}$ by using the lifting principle ([1]). Moreover, if, for example, $k \leq n$, then in the super-critical case, we can replace $R_{k}$ by the operator of multiplication $t^{k / n} g(t)$. This implies a very simple characterization of both optimal target space $H$ and optimal domain space $E$. Namely, the quasinorm in the optimal target space $H(E)$ is given by $\rho_{E}\left(t^{-k / n} g(t)\right)$ and the quasinorm in the optimal domain space $E(H)$ is given by $\rho_{H}\left(t^{k / n} g(t)\right)$. Note that we do not require $\rho_{E}$ to be rearrangement invariant. In the critical case, the formula for the optimal target space is more complicated. In some cases it can be simplified. To this end, we apply the $\Sigma^{q}$ method of extrapolation ([3]) from the super-critical case. As a byproduct, we also characterize the embedding $W^{k} E \hookrightarrow$ $C^{j}, j<k$, where $C^{j}$ consists of all functions with bounded and uniformly continuous derivatives up to order $j$. Namely, this is equivalent to the embedding $E \hookrightarrow L^{n /(k-j), 1}$ if $k \leq n$. The embedding $W^{n+j} E \hookrightarrow C^{j}$ is always true since $W^{n} E \hookrightarrow$ $W_{1}^{n} \hookrightarrow C^{0}$.

The problem of the optimal target rearrangement invariant space for potential type operators is considered in [4] by using $L^{p}$-capacities. The problem of the mapping properties of the Riesz potential in optimal couples of rearrangement invariant spaces is treated in [5-7]. The optimal embeddings of generalized Sobolev type spaces into rearrangement invariant spaces are characterized in several papers $[5,8-$ 21]. The characterization of the continuous embedding of the generalized Bessel potential spaces into the generalized Hölder-Zygmund spaces $\mathscr{C H}$, when $H$ is a weighted Lebesgue space, is given in [22]. The optimal embeddings of Calderón spaces into the generalized Hölder-Zygmund spaces are characterized in [23].

The plan of the paper is as follows. In Section 2 we provide some basic definitions and known results. In Section 3 we characterize the embedding $W^{k} E \hookrightarrow \mathscr{C H}$. The optimal quasinorms are constructed in Section 4 .

\section{Preliminaries}

We use the notations $a_{1} \leqslant a_{2}$ or $a_{2} \gtrsim a_{1}$ for nonnegative functions or functionals to mean that the quotient $a_{1} / a_{2}$ is bounded; also, $a_{1} \approx a_{2}$ means that $a_{1} \lesssim a_{2}$ and $a_{1} \gtrsim a_{2}$. We say that $a_{1}$ is equivalent to $a_{2}$ if $a_{1} \approx a_{2}$.

Let $E$ be a quasinormed rearrangement invariant space as in the Introduction. There is an equivalent quasinorm $\rho_{p} \approx \rho_{E}$ that satisfies the triangle inequality $\rho_{p}^{p}\left(g_{1}+g_{2}\right) \leq \rho_{p}^{p}\left(g_{1}\right)+$ $\rho_{p}^{p}\left(g_{2}\right)$ for some $p \in(0,1]$ that depends only on the space $E$ (see [24]). We say that the quasinorm $\rho_{E}$ satisfies Minkowski's inequality if for the equivalent quasinorm $\rho_{p}$,

$$
\rho_{p}^{p}\left(\sum g_{j}\right) \lesssim \sum \rho_{p}^{p}\left(g_{j}\right), \quad g_{j} \in M^{+}
$$

Usually we apply this inequality for functions $g_{j} \in M^{+}$with some kind of monotonicity.

Recall the definition of the lower and upper Boyd indices $\alpha_{E}$ and $\beta_{E}$. Let $g_{u}(t)=g(t / u)$ if $t<u$ and $g_{u}(t)=0$ if $t \geq u$, where $0<t<1, g \in M^{+}$, and let

$$
\begin{aligned}
& h_{E}(u)=\sup \left\{\frac{\rho_{E}\left(g_{u}^{*}\right)}{\rho_{E}\left(g^{*}\right)}: g \in M^{+}\right\}, \\
& g_{u}(t):=g\left(\frac{t}{u}\right), \quad u>0,
\end{aligned}
$$

be the dilation function generated by $\rho_{E}$. Suppose that it is finite. Then

$$
\alpha_{E}:=\sup _{0<t<1} \frac{\log h_{E}(t)}{\log t}, \quad \beta_{E}:=\inf _{1<t<\infty} \frac{\log h_{E}(t)}{\log t} .
$$

The function $h_{E}$ is submultiplicative, increasing, $h_{E}(1)=$ $1, h_{E}(u) h_{E}(1 / u) \geq 1$; hence $0 \leq \alpha_{E} \leq \beta_{E}$. We suppose that $0<\alpha_{E}=\beta_{E} \leq 1$.

If $\beta_{E}<1$ we have by using Minkowski's inequality that $\rho_{E}\left(f^{*}\right) \approx \rho_{E}\left(f^{* *}\right)$. In particular, $\|f\|_{E} \approx \rho_{E}\left(f^{* *}\right)$ if $\beta_{E}<$ 1. For example, consider the Gamma spaces $E=\Gamma^{q}(w)$, $0<q \leq \infty$, $w$-positive weight, that is, a positive function from $M^{+}$, with a quasinorm $\|f\|_{\Gamma^{q}(w)}:=\rho_{E}\left(f^{*}\right), \rho_{E}(g):=$ $\rho_{w, q}\left(\int_{0}^{1} g(t u) d u\right)$, where

$$
\begin{aligned}
\rho_{w, q}(g):= & \left(\int_{0}^{1}[g(t) w(t)]^{q} \frac{d t}{t}\right)^{1 / q}, \quad g \in M^{+}, \\
& \left(\int_{0}^{1} w^{q}(t) \frac{d t}{t}\right)^{1 / q}<\infty .
\end{aligned}
$$

Then $L^{\infty}(\Omega) \hookrightarrow \Gamma^{q}(w) \hookrightarrow L^{1}(\Omega)$. If $w(t)=t^{1 / p}, 1<p<\infty$, we write as usual $L^{p, q}$ instead of $\Gamma^{q}\left(t^{1 / p}\right)$. Consider also the classical Lorentz spaces $\Lambda^{q}(w), 0<q \leq \infty$; $f \in \Lambda^{q}(w)$ if $\|f\|_{\Lambda_{w}^{q}}:=\rho_{w, q}\left(f^{*}\right)<\infty, w(2 t) \approx w(t)$. We suppose that $L^{\infty}(\Omega) \hookrightarrow \Lambda^{q}(w) \hookrightarrow L^{1}(\Omega)$.

Note that if $E=\Lambda^{q}\left(t^{\alpha} w\right), 0<\alpha \leq 1$, where $w$ is slowly varying, then $\alpha_{E}=\beta_{E}=\alpha$. Recall that $w \in M^{+}$is slowly varying if for all $\varepsilon>0$ the function $t^{\varepsilon} w(t)$ is equivalent to an increasing function and the function $t^{-\varepsilon} w(t)$ is equivalent to a decreasing function.

In order to introduce the Hölder-Zygmund class of spaces, we denote the modulus of continuity of order $k$ by

$$
\omega^{k}(t, f)=\sup _{|h| \leq t} \sup _{x \in \mathbf{R}^{n}}\left|\Delta_{h}^{k} f(x)\right|,
$$

where $\Delta_{h}^{k} f$ are the usual iterated differences of $f$. When $k=1$ we simply write $\omega(t, f)$.

Let $H$ be a quasinormed space of locally integrable functions on the interval $(0,1)$ with the Lebesgue measure, continuously embedded in $L^{\infty}(0,1)$ and $\|g\|_{H}=\rho_{H}(|g|)$, where $\rho_{H}$ is a monotone quasinorm on $M^{+}$which satisfies Minkowski's inequality. The dilation function generated by $\rho_{H}$ is given by

$$
h_{H}(u)=\sup \left\{\frac{\rho_{H}\left(g_{1 / u}\right)}{\rho_{H}(g)}: g \in M_{m}\right\},
$$


where

$$
\begin{aligned}
M_{m}:=\left\{g \in M^{+}: g(t)\right. \text { is increasing, } \\
\left.\quad t^{-m / n} g(t) \text { is decreasing and } g(2 t) \approx g(t)\right\} .
\end{aligned}
$$

The choice of the space $M_{m}$ is motivated by the fact that $\omega^{m}\left(t^{1 / n}, f\right)$ is equivalent to a function $g \in M_{m}$. The function $h_{H}(u)$ is submultiplicative, increasing and $u^{-m / n} h_{H}(u)$ is decreasing and $h_{H}(1)=1, h_{H}(u) h_{H}(1 / u) \geq 1$. Therefore the Boyd indices of $H$ are well defined

$$
\alpha_{H}=\sup _{0<t<1} \frac{\log h_{H}(t)}{\log t}, \quad \beta_{H}=\inf _{1<t<\infty} \frac{\log h_{H}(t)}{\log t},
$$

and they satisfy $\alpha_{H} \leq \beta_{H} \leq m / n$. In what follows, we suppose that $0 \leq \alpha_{H}=\beta_{H} \leq m / n$.

For example, let $H=L_{*}^{q}\left(b(t) t^{-\gamma / n}\right)$. Here $0 \leq \gamma \leq m$ and $b$ is a slowly varying function, and $L_{*}^{q}(w)$, or simply $L_{*}^{q}$ if $w=1$, is the weighted Lebesgue space with a quasinorm $\|g\|_{L_{*}^{q}(w)}=\rho_{w, q}(|g|)$, where $\rho_{w, q}$ is given by (5). It turns out that $\alpha_{H}=\beta_{H}=\gamma / n$.

Definition 1. Let $j=0,1, \ldots$ and let $C^{j}$ stand for the space of all functions $f$, defined on $\mathbf{R}^{n}$, that have bounded and uniformly continuous derivatives up to the order $j$, normed by $\|f\|_{C^{j}}=\sup \sum_{l=0}^{j}\left|P^{l} f(x)\right|$, where $P^{l} f(x)=\sum_{|v|=l} D^{\nu} f(x)$.

(i) If $j / n<\alpha_{H}<(j+1) / n$ for $j \geq 1$ or $0 \leq \alpha_{H}<1 / n$ for $j=0$, then $\mathscr{C H}$ is formed by all functions $f$ in $C^{j}$ having a finite quasinorm

$$
\|f\|_{\mathscr{C} H}=\|f\|_{C^{j}}+\rho_{H}\left(\chi_{(0,1)}(t) t^{j / n} \omega\left(t^{1 / n}, P^{j} f\right)\right) .
$$

(ii) If $\alpha_{H}=(j+1) / n$, then $\mathscr{C} H$ consists of all functions $f$ in $C^{j}$ having a finite quasinorm

$$
\|f\|_{\mathscr{C} H}=\|f\|_{C^{j}}+\rho_{H}\left(\chi_{(0,1)}(t) t^{j / n} \omega^{2}\left(t^{1 / n}, P^{j} f\right)\right) .
$$

Here $\chi_{(a, b)}, 0<a<b<\infty$, is the characteristic function of the interval $(a, b)$.

In particular, if $H=L^{\infty}\left(t^{-\gamma / n}\right), \gamma>0$, then $\mathscr{C} H$ coincides with the usual Hölder-Zygmund space $\mathscr{C}^{\gamma}$ (see [1]). Also, if $H=L^{\infty}$, then $\mathscr{C} H=C^{0}$.

We will use the following equivalent quasinorm.

Theorem 2 (equivalence [23]). Let $0 \leq \alpha_{H}=\beta_{H}<m / n$. If $\rho_{H}\left(\chi_{(0,1)}(t) t^{\alpha}\right)<\infty$ for $\alpha>\alpha_{H}$, then for all such $m$,

$$
\|f\|_{\mathscr{C} H} \approx\|f\|_{C^{0}}+\rho_{H}\left(\chi_{(0,1)}(t) \omega^{m}\left(t^{1 / n}, f\right)\right) .
$$

Note that if $\rho_{H}\left(\chi_{(0,1)}(t) t^{m / n}\right)<\infty$, then $\mathscr{C H}$ is a $K$ interpolation space for the couple $\left(C^{0}, C^{m}\right)$, namely, $\mathscr{C H}=$ $\left(C^{0}, C^{m}\right)_{H_{1}}$, where $\rho_{H_{1}}(g)=\rho_{H}\left(g\left(t^{m / n}\right)\right)$. In particular, $\mathscr{C} L_{*}^{1}\left(t^{-j}\right) \hookrightarrow C^{j} \hookrightarrow \mathscr{C} L^{\infty}\left(t^{-j}\right)$. By $\chi_{(a, b)}, 0 \leq a<b \leq \infty$ we denote the characteristic function of the interval $(a, b)$.
Recall some basic definitions from the theory of interpolation spaces [21]. Let $\left(A_{0}, A_{1}\right)$ be a couple of two quasinormed spaces, such that both are continuously embedded in some quasinormed space and let

$$
\begin{aligned}
K(t, f) & =K\left(t, f ; A_{0}, A_{1}\right) \\
& =\inf _{f=f_{0}+f_{1}}\left\{\left\|f_{0}\right\|_{A_{0}}+t\left\|f_{1}\right\|_{A_{1}}\right\}, \quad f \in A_{0}+A_{1},
\end{aligned}
$$

be the $K$-functional of Peetre. By definition, the $K$-interpolation space $A_{\Phi}=\left(A_{0}, A_{1}\right)_{\Phi}$ has a quasinorm $\|f\|_{A_{\Phi}}=$ $\|K(t, f)\|_{\Phi}$, where $\Phi$ is a quasinormed function space with a monotone quasinorm on $(0, \infty)$ with the Lebesgue measure and such that $\min \{1, t\} \in \Phi$. Then $A_{0} \cap A_{1} \hookrightarrow A_{\Phi} \hookrightarrow$ $A_{0}+A_{1}$. If

$$
\begin{gathered}
\|g\|_{\Phi}=\left(\int_{0}^{\infty}\left[w(t) t^{-\theta} g(t)\right]^{q} \frac{d t}{t}\right)^{1 / q}, \\
0 \leq \theta \leq 1, \quad 0<q \leq \infty, \quad w \in \mathscr{M}^{+},
\end{gathered}
$$

we write $\left(A_{0}, A_{1}\right)_{w t^{-\theta}, q}$ instead of $\left(A_{0}, A_{1}\right)_{\Phi}$. Also, if $w=1$ then we write $\left(A_{0}, A_{1}\right)_{\theta, q}$. By definition,

$$
\begin{aligned}
& \|f\|_{A_{0} \cap A_{1}}=\|f\|_{A_{0}}+\|f\|_{A_{1}}, \\
& \|f\|_{A_{0}+A_{1}}=K\left(1, f ; A_{0}, A_{1}\right) .
\end{aligned}
$$

Theorem 3 (lifting principle). Let $\alpha_{H}>0$ and let $\rho_{H_{j}}(g):=$ $\rho_{H}\left(t^{-j / n} g(t)\right), j \geq 1$. Then

$$
\|f\|_{\mathscr{C} H_{j}} \approx\left\|\mathscr{D}^{j} f\right\|_{\mathscr{C} H^{\prime}}, \quad \mathscr{D}^{j}=\sum_{|\alpha| \leq j} D^{\alpha} .
$$

Proof. Let $m / n>\alpha_{H}+j / n$. Since

$$
\omega^{m}\left(t^{1 / n}, f\right) \lesssim t^{j / n} \omega^{m-j}\left(t^{1 / n}, P^{j} f\right),
$$

it follows

$$
\rho_{H_{j}}\left(\omega^{m}\left(t^{1 / n}, f\right)\right) \lesssim \rho_{H}\left(\omega^{m-j}\left(t^{1 / n}, \mathscr{D}^{j} f\right)\right) .
$$

Hence

$$
\|f\|_{\mathscr{C} H_{j}} \leqslant\left\|\mathscr{D}^{j} f\right\|_{\mathscr{C} H^{\prime}} .
$$

To prove the reverse, we use the formula (see [25, page 342])

$$
\omega^{m}\left(t^{1 / n}, D^{\alpha} f\right) \lesssim \int_{0}^{t} u^{-|\alpha| / n} \omega^{m}\left(u^{1 / n}, f\right) \frac{d u}{u} .
$$

Then applying Minkowski's inequality and $\alpha_{H_{j}}=\beta_{H_{j}}=j / n+$ $\alpha_{H}>j / n$, we get

$$
\begin{array}{r}
\rho_{H}\left(\chi_{(0,1)}(t) \omega^{m}\left(t^{1 / n}, D^{\alpha} f\right)\right) \leqslant \rho_{H_{j}}\left(\chi_{(0,1)}(t) \omega^{m}\left(t^{1 / n}, f\right)\right), \\
|\alpha| \leq j .
\end{array}
$$

Since $\mathscr{C} H_{j} \hookrightarrow C^{j}$, we derive

$$
\left\|\mathscr{D}^{j} f\right\|_{\mathscr{C} H} \leqslant\|f\|_{\mathscr{C} H_{j}} .
$$


Remark 4. The relation (19) is always true. But if $\alpha_{H}=0$ then the reverse might not be true. For example, let $n=1, H=L^{\infty}$, $j=1$, and $f(x)=x \ln |2 x|$ if $|x| \leq 1 / 2$ and $f(x)=0$ if $|x|>1 / 2$. Then $f \notin C^{1}$, but $\omega^{2}(t, f)=O(t)$.

It will be convenient to introduce the classes of the domain and target quasinorms, where the optimality is investigated. Let $N_{d}$ consist of all domain quasinorms $\rho_{E}$ that are monotone, satisfying Minkowski's inequality, $0<\alpha_{E}=$ $\beta_{E} \leq \min (k, n) / n, \rho_{E}\left(\chi_{(0,1)} t^{-\alpha}\right)<\infty$ if $\alpha<\alpha_{E}$ and the condition (30) below for $k \leq n$, and $E \hookrightarrow L^{1}$ for $k>n$. Let $N_{t}$ consist of all target quasinorms $\rho_{H}$ that are monotone, satisfy Minkowski's inequality, $0 \leq \alpha_{H}=\beta_{H}<\min (k, n) / n$, $\rho_{H}\left(\chi_{(0,1)}(t) t^{\alpha}\right)<\infty$ if $\alpha>\alpha_{H}$ and

$$
\sup _{0<t<1} g(t) \lesssim \rho_{H}(g) \text {. }
$$

We use the following definitions.

Definition 5 (admissible couple). We say that the couple $\rho_{E} \in$ $N_{d}, \rho_{H} \in N_{t}$ is admissible if $W^{k} E \hookrightarrow \mathscr{C H}$ when $k \leq n$, and if $\mathscr{D}^{j}\left(W^{n+j} E\right) \hookrightarrow \mathscr{C} H$ for $j \geq 1$. Moreover, $\rho_{E}(E)$ is called domain quasinorm (domain space), and $\rho_{H}(H)$ is called target quasinorm (target space).

Definition 6 (optimal target quasi-norm). Given the domain quasinorm $\rho_{E} \in N_{d}$, the optimal target quasi-norm, denoted by $\rho_{H(E)}$, is the strongest target quasi-norm; that is,

$$
\rho_{H}(g) \lesssim \rho_{H(E)}(g), \quad g \in M_{\min (k, n)}
$$

for any target quasinorm $\rho_{H} \in N_{t}$ such that the couple $\rho_{E}$, $\rho_{H}$ is admissible. Since $\mathscr{C H}(E) \hookrightarrow \mathscr{C H}$, we call $\mathscr{C H}(E)$ the optimal Hölder-Zygmund space.

Definition 7 (optimal domain quasi-norm). Given the target quasinorm $\rho_{H} \in N_{t}$, the optimal domain quasi-norm, denoted by $\rho_{E(H)}$, is the weakest domain quasi-norm; that is,

$$
\rho_{E(H)}\left(f^{*}\right) \lesssim \rho_{E}\left(f^{*}\right), \quad f \in L^{1}(\Omega),
$$

for any domain quasinorm $\rho_{E} \in N_{d}$ such that the couple $\rho_{E}$, $\rho_{H}$ is admissible.

Definition 8 (optimal couple). The admissible couple $\rho_{E} \in$ $N_{d}, \rho_{H} \in N_{t}$ is said to be optimal if both $\rho_{E}$ and $\rho_{H}$ are optimal.

\section{Admissible Couples}

Here we give a characterization of all admissible couples $\rho_{E} \epsilon$ $N_{d}, \rho_{H} \in N_{t}$. We start with the main estimate. For $k=1$, see also [26].

Theorem 9. Let $f \in W_{1}^{k}$ and $k \leq n$. Then

$$
\omega^{k}\left(t^{1 / n}, f\right) \lesssim \int_{0}^{t} u^{k / n-1}\left(\mathscr{D}^{k} f\right)^{*}(u) d u
$$

where $\mathscr{D}^{k} f:=\sum_{|\alpha| \leq k} D^{\alpha} f$.
Proof. We use the embedding

$$
W^{k} L^{n / k, 1} \hookrightarrow L^{\infty}
$$

whence

$$
\begin{aligned}
\omega^{k}\left(t^{1 / n}, f\right) & \lesssim K\left(t^{k / n}, f ; L^{\infty}, W_{\infty}^{k}\right) \\
& \lesssim K\left(t^{k / n}, f ; W^{k} L^{n / k, 1}, W_{\infty}^{k}\right) .
\end{aligned}
$$

Then (26) follows from the basic formula [25, page 360]

$$
K\left(t, f ; W_{1}^{k}, W_{\infty}^{k}\right) \approx \int_{0}^{t}\left(\mathscr{D}^{k} f\right)^{*}(s) d s
$$

and the reiteration formula of Holmstedt for the $K$-functional [25, page 310].

Now we discuss the embedding $W^{k} E \hookrightarrow C^{0}$. For $k=1$ more general results are proved in [27, Chapter 4].

Theorem 10. A necessary and sufficient condition for the embedding $W^{k} E \hookrightarrow C^{0}, k \leq n$, is the following one

$$
\int_{0}^{1} t^{k / n-1} g(t) d t \lesssim \rho_{E}(g), \quad g \in D_{1},
$$

where

$$
D_{1}=\left\{g \in M^{+}: g \text { is decreasing function }\right\} .
$$

Proof. The conditions (30) and (26), (27) imply the embed$\operatorname{ding} W^{k} E \hookrightarrow L^{\infty}$ and $\lim _{t \rightarrow 0} \omega^{k}\left(t^{1 / n}, f\right)=0$ if $f \in W^{k} E$. On the other hand, by Marchaud's inequality (see [25], Theorem 5.4.4), we have

$$
\omega\left(t^{1 / n}, f\right) \lesssim t^{1 / n} \int_{t}^{\infty} u^{-1 / n} \omega^{k}\left(u^{1 / n}, f\right) \frac{d u}{u} .
$$

It is easy to see that $\lim _{t \rightarrow 0} \omega\left(t^{1 / n}, f\right)=0$. Thus $W^{k} E \hookrightarrow C^{0}$.

Before proving the reverse, note that (30) is always satisfied if $k / n>\alpha_{E}$. Since

$$
\rho_{E}\left(\chi_{(0,1)}\right) g(u) \leq \rho_{E}(g(t u)) \leq h_{E}\left(\frac{1}{u}\right) \rho_{E}(g),
$$

we have

$$
g(u) \lesssim h_{E}\left(\frac{1}{u}\right) \rho_{E}(g), \quad g \in D_{1} .
$$

Hence for $0<\varepsilon<k / n-\alpha_{E}$,

$$
\int_{0}^{1} u^{k / n-1} g(u) d u \lesssim \rho_{E}(g) \int_{0}^{1} u^{k / n-\alpha_{E}-\varepsilon} \frac{d u}{u} \lesssim \rho_{E}(g) .
$$

It remains to prove that if $W^{k} E \hookrightarrow C^{0}, k \leq n$, then (30) is true for $\alpha_{E}=k / n$. To this end we choose a test function $f$ as follows:

$$
f(x)=\int_{0}^{1} g(u) u^{k / n} \psi\left(x u^{-1 / n}\right) \frac{d u}{u},
$$


where $g \in D_{1}$ and $\psi$ is in $C_{0}^{\infty}$ such that $\psi(x)=1$ if $|x| \leq$ $2^{-1} c^{-1 / n}$ and $\psi(x)=0$ if $|x| \geq c^{-1 / n}$. Then $f(x)=0$ for $|x|>$ $c^{-1 / n}$, whence $f^{*}(t)=0$ for $t>1$ for appropriate $c$ and $|f(x)| \leq \int_{c|x|^{n}}^{1} g(u) u^{k / n} d u / u$, whence $|f(x)| \lesssim g\left(c|x|^{n}\right) ;$ therefore $f^{*}(t) \lesssim g(t)$. This implies

$$
\rho_{E}\left(f^{*}\right) \lesssim \rho_{E}(g) .
$$

Analogously, since $\left|D^{\alpha} f(x)\right| \leq \int_{c|x|^{n}}^{2^{n} c|x|^{n}} g(u) d u / u, 1 \leq|\alpha| \leq k$, we have

$$
\|f\|_{W^{k} E} \leqslant \rho_{E}(g) .
$$

Also $f(0)=\psi(0) / n \int_{0}^{1} u^{k / n-1} g(u) d u \lesssim\|f\|_{W^{k} E} \lesssim \rho_{E}(g)$. Thus (30) is proved.

Remark 11. Similar arguments show that $W^{k} E \hookrightarrow C^{j}, j<$ $k \leq n$, if and only if $E \hookrightarrow L^{n /(k-j), 1}$.

Theorem 12. The couple $\rho_{E} \in N_{d}, \rho_{H} \in N_{t}$ is admissible if and only if

$$
\rho_{H}\left(\chi_{(0,1)} R_{\min (k, n)} g\right) \lesssim \rho_{E}(g), \quad g \in D_{1},
$$

where

$$
R_{k} g(t):=\int_{0}^{t} u^{k / n-1} g(u) d u .
$$

Proof. Step 1 (sufficiency of (39)). If $k \leq n$ then it is clear that the embedding $W^{k} E \hookrightarrow \mathscr{C H}$ follows from (39), (26), and (27). Let now $k=n+j, j \geq 1$. Then (39) for $k=n$ implies $W^{n} E \hookrightarrow \mathscr{C} H$. Hence $\mathscr{D}^{j}\left(W^{n+j} E\right) \hookrightarrow \mathscr{C} H$ for $j \geq 1$.

Step 2 (necessity of (39) when $k \leq n$ ). Now we prove that the embedding $W^{k} E \hookrightarrow \mathscr{C} H$ implies (39) for $k \leq n$. To this end we choose the test function $f$ as in (36).

Let $|h|=C t^{1 / n}$. We split $f=f_{1 t}+f_{2 t}$, $f_{1 t}(x)=\int_{0}^{t} u^{k / n} g(u) \psi\left(x u^{-1 / n}\right) d u / u, \quad f_{2 t}(x)=$ $\int_{t}^{1} u^{k / n} g(u) \psi\left(x u^{-1 / n}\right) d u / u, 0<t<1$. First we prove that for some large $C>0$,

$$
\omega^{m}\left(C t^{1 / n}, f_{1 t}\right) \geq \psi(0) R_{k} g(t), \quad 0<t<1 .
$$

Indeed, we have $\omega^{m}\left(C t^{1 / n}, f_{1 t}\right) \geq\left|\left(\Delta_{h}^{m} f_{1 t}\right)(0)\right|$ and $\psi\left(j C t^{1 / n} u^{-1 / n}\right)=0$ for $u<t, 1 \leq j \leq m$ if $C>0$ is large enough. Hence (41) follows. Further,

$$
\omega^{m}\left(t^{1 / n}, f\right) \geq \omega^{m}\left(t^{1 / n}, f_{1 t}\right)-\omega^{m}\left(t^{1 / n}, f_{2 t}\right), \quad 0<t<1 .
$$

Since

$$
\begin{aligned}
\omega^{m}\left(t^{1 / n}, f_{2 t}\right) & \lesssim t^{m / n}\left\|P^{m} f_{2 t}\right\|_{L^{\infty}} \\
& \lesssim t^{m / n} \int_{t}^{1} u^{(k-m) / n} g(u) \frac{d u}{u}
\end{aligned}
$$

and $R_{k} g(t) \gtrsim t^{k / n} g(t)$ for $g \in D_{1}$, we get

$$
\omega^{m}\left(t^{1 / n}, f_{2 t}\right) \leqslant t^{m / n} \int_{t}^{1} u^{-m / n} R_{k} g(u) \frac{d u}{u} .
$$

Therefore

$$
\begin{array}{r}
R_{k} g(t) \leq c_{1} \omega^{m}\left(t^{1 / n}, f\right)+c t^{m / n} \int_{t}^{1} u^{-m / n} R_{k} g(u) \frac{d u}{u}, \\
0<t<1, \\
R_{k} g(t) \leq c_{1} \omega^{m}\left(t^{1 / n}, f\right)+c t^{m / n} \int_{t}^{1} u^{-m / n} g(u) \frac{d u}{u}, \\
0<t<1 .
\end{array}
$$

To solve the integral inequality (45) for $p(t)$ := $\chi_{(0,1)}(t) t^{-m / n} R_{k} g(t)$, we set $q(t)=c_{1} t^{-m / n} \omega^{m}\left(t^{1 / n}, f\right)$ and rewrite it as $p(t) \leq q(t)+c \int_{t}^{1} p(u) d u / u$. If $r(t)=\int_{t}^{1} p(u) d u / u$, then we get the differential inequality $0 \leq \operatorname{tr}^{\prime}(t)+\operatorname{cr}(t)+q(t)$. If $r(t)=t^{-c} v(t)$, then $0 \leq v^{\prime}(t)+t^{c-1} q(t)$, whence $v(t) \leq \int_{t}^{1} u^{c-1} q(u) d u$. Therefore

$$
\chi_{(0,1 / 2)}(t) R_{k} g(t) \lesssim t^{m / n-c} \int_{t}^{1} u^{c-m / n} \omega^{m}\left(u^{1 / n}, f\right) \frac{d u}{u} .
$$

Hence by using Minkowski's inequality and choosing $m$ large enough, we obtain

$$
\rho_{H}\left(\chi_{(0,1 / 2)} R_{k} g\right) \lesssim \rho_{H}\left(\omega^{m}\left(t^{1 / n}, f\right)\right) .
$$

On the other hand, from (46), it follows that

$$
\rho_{H}\left(\chi_{(1 / 2,1)} R_{k} g\right) \leq \rho_{H}\left(\omega^{m}\left(t^{1 / n}, f\right)\right)+\int_{0}^{1} g(u) d u .
$$

Hence, using also (30), we get

$$
\rho_{H}\left(\chi_{(0,1)} R_{k} g\right) \lesssim \rho_{H}\left(\omega^{m}\left(t^{1 / n}, f\right)\right)+\rho_{E}(g) .
$$

Thus, if $W^{k} E \hookrightarrow \mathscr{C} H$ is given, then (50), (38) imply (39).

Step 3 (necessity of (39) when $k=n+j, j \geq 1$ ). Now we prove that the embedding $\mathscr{D}^{j}\left(W^{n+j} E\right) \hookrightarrow \mathscr{C} H, j \geq 1$, implies (39) for $k=n$. To this end we choose the test function $f$ in the form

$$
f(x)=\int_{0}^{1} g(u) u^{j / n} \psi\left(x u^{-1 / n}\right) d u,
$$

where $g \in D_{1}$ and $\psi$ is the same as in $(36)$. Note that $\mathscr{D}^{j} \psi(0)=$ 1 . Then as before we get (37), and

$$
\left|D^{\alpha} f(x)\right| \lesssim g\left(c|x|^{n}\right)
$$

for $1 \leq|\alpha| \leq n+j$. Hence

$$
\|f\|_{W^{n+j} E} \lesssim \rho_{E}(g) .
$$


On the other hand, using the arguments from Step 2 but for the function $\mathscr{D}^{j} f, j \geq 1$, we obtain

$$
\rho_{H}\left(\chi_{(0,1)} R_{n} g\right) \lesssim \rho_{H}\left(\omega^{m}\left(t^{1 / n}, \mathscr{D}^{j} f\right)\right)+\rho_{E}(g), \quad j \geq 1 .
$$

Thus, if $\mathscr{D}^{j}\left(W^{n+j} E\right) \hookrightarrow \mathscr{C} H, j \geq 1$ is given, then (54), (53) imply (39) for $k=n$.

Theorem 13. Let $\alpha_{H}>0$. Then the couple $\rho_{E} \in N_{d}, \rho_{H} \in N_{t}$ is admissible for $k>n$ if and only if

$$
W^{n+j} E \hookrightarrow \mathscr{C} H_{j}, \quad \rho_{H_{j}}(g):=\rho_{H}\left(t^{-j / n} g(t)\right), \quad j \geq 1 .
$$

Moreover, (55) is equivalent to

$$
\rho_{H_{j}}\left(R_{n+j} g\right) \lesssim \rho_{E}(g), \quad g \in D_{1} \text {. }
$$

Proof. Let $\rho_{E} \in N_{d}, \rho_{H} \in N_{t}$ be an admissible couple for $k>n$. Then $\mathscr{D}^{j}\left(W^{n+j} E\right) \hookrightarrow \mathscr{C} H$. Since

$$
\omega^{m}\left(t^{1 / n}, \mathscr{D}^{j} f\right) \lesssim \int_{0}^{t}\left(\mathscr{D}^{n+j} f\right)^{*}(u) d u, \quad m \geq n,
$$

we have, by applying (39) for $k=n$,

$$
\rho_{H}\left(\omega^{m}\left(t^{1 / n}, \mathscr{D}^{j} f\right)\right) \lesssim \rho_{E}\left(\left(\mathscr{D}^{n+j} f\right)^{*}\right) .
$$

Using also (16) and $W^{n+j} E \hookrightarrow C^{j}$, we obtain $W^{n+j} E \hookrightarrow$ $\mathscr{C} H_{j}$. Further, as in the proof of the previous theorem, this embedding implies (56). Finally,

$$
\rho_{H_{j}}\left(R_{n+j} g\right) \approx \rho_{H}\left(R_{n} g\right), \quad \alpha_{H}>0 .
$$

Indeed, $\rho_{H}\left(R_{n} g\right)=\rho_{H_{j}}\left(t^{j / n} R_{n} g(t)\right)$ and

$$
\begin{aligned}
\rho_{H_{j}}\left(t^{j / n} R_{n} g(t)\right) & \\
& \leqslant \rho_{H_{j}}\left(t^{j / n} \int_{0}^{t} u^{(n+j) / n}\left(R_{n+j} g\right)(u) d u\right) .
\end{aligned}
$$

Applying Minkowski’s inequality, we get, since $\alpha_{H_{j}}>j / n$,

$$
\rho_{H}\left(R_{n} g\right) \lesssim \rho_{H_{j}}\left(R_{n+j} g\right)
$$

For the reverse, we notice that $R_{n+j} g(t) \lesssim t^{j / n} R_{n} g(t)$. Then

$$
\rho_{H_{j}}\left(R_{n+j} g\right) \lesssim \rho_{H}\left(R_{n} g\right) .
$$

\section{Optimal Quasinorms}

Here we give a characterization of the optimal domain and optimal target quasinorms.
4.1. Optimal Domain Quasinorms. We can construct an optimal domain quasinorm $\rho_{E(H)}$ by Theorem 9 as follows.

Definition 14 (construction of an optimal domain quasinorm). For a given target quasinorm $\rho_{H} \in N_{t}$, we set

$$
\rho_{E(H)}(g):=\rho_{H}\left(R_{\min (k, n)} g\right), \quad g \in M^{+} .
$$

Note that $R_{\min (k, n)}\left(g_{u}\right)=u^{\min (k, n) / n}\left(\widetilde{R_{\min (k, n)}} g\right)_{1 / u}$ and $S g \in L$ if $g \in D_{1}$. Hence $\alpha_{E(H)}=\beta_{E(H)}=\min (k, n) / n-\alpha_{H}$.

Theorem 15. The quasinorm $\rho_{E(H)}$ belongs to $N_{d}$, the couple $\rho_{E(H)}, \rho_{H}$ is admissible, and the domain quasinorm $\rho_{E(H)}$ is optimal. Moreover, the target quasinorm $\rho_{H}$ is also optimal and

$$
\rho_{E(H)}(g) \approx \rho_{H}\left(t^{\min (k, n) / n} g\right), \quad g \in D_{1} \text { if } \alpha_{H}>0 .
$$

Proof. It is easy to check that $\rho_{E(H)} \in N_{d}$. Further, the couple $\rho_{E(H)}, \rho_{H}$ is admissible since $\rho_{H}\left(R_{\min (k, n)} g\right)=\rho_{E(H)}(g)$, $g \in D_{1}$. Moreover, $\rho_{E(H)}$ is optimal, since for any admissible couple $\rho_{E_{1}} \in N_{d}, \rho_{H}$, we have $\rho_{H}\left(\chi_{(0,1)} R_{\min (k, n)} g\right) \leqslant \rho_{E_{1}}(g)$, where $g \in D_{1}$. Therefore for $f \in L^{1}(\Omega)$,

$$
\begin{aligned}
\rho_{E(H)}\left(f^{*}\right) & =\rho_{H}\left(R_{\min (k, n)} f^{*}\right) \\
& \lesssim \rho_{H}\left(\chi_{(0,1)} R_{\min (k, n)} f^{*}\right)+\rho_{E_{1}}\left(f^{*}\right) \lesssim \rho_{E_{1}}\left(f^{*}\right) .
\end{aligned}
$$

To prove that $\rho_{H}$ is also optimal, let $\rho_{E(H)}, \rho_{H_{1}} \in N_{t}$ be an arbitrary admissible couple. Then

$$
\rho_{H_{1}}\left(\chi_{(0,1)} R_{\min (k, n)} g\right) \lesssim \rho_{E(H)}(g), \quad g \in D_{1} .
$$

It is enough to check that

$$
\rho_{H_{1}}\left(\chi_{(0,1)} g\right) \lesssim \rho_{H}(g), \quad g \in M_{\min (k, n)} .
$$

Let $k<n$. (The case $k \geq n$ is easier.) We introduce a better function $g_{1}(t)=g\left(t^{n / k}\right)$. Then $g_{1}$ is quasiconcave; therefore $g_{1}(t) \approx \int_{0}^{t} h_{1}(u) d u$ and $h_{1} \in D_{1}$. By changing the variables, we get $g \approx R_{k} h$ with $h \in D_{1}$. Then

$$
\begin{aligned}
\rho_{H_{1}}\left(\chi_{(0,1)} g\right) & \lesssim \rho_{H_{1}}\left(\chi_{(0,1)} R_{k} h\right) \\
& \lesssim \rho_{E(H)}(h) \approx \rho_{H}\left(R_{k} h\right) \approx \rho_{H}(g) .
\end{aligned}
$$

Thus (67) is proved. To prove the equivalence (64), we use $t^{\min (k, n) / n} g(t) \lesssim R_{\min (k, n)} g(t), g \in D_{1}$ and Minkowski's inequality as follows:

$$
\begin{array}{r}
\rho_{H}^{p}\left(R_{\min (k, n)} g\right) \lesssim \sum_{j=-\infty}^{0} h_{H}^{p}\left(2^{j}\right) \rho_{H}^{p}\left(t^{\min (k, n) / n} g(t)\right), \\
g \in D_{1}, \alpha_{H}>0,
\end{array}
$$

whence $\rho_{E(H)}(g) \lesssim \rho_{H}\left(t^{\min (k, n) / n} g(t)\right), g \in D_{1}$.

Remark 16. Let $k \geq n$ and $\chi_{(0,1)}(t) t \in H$. Then the couple $E=\left(L^{1}, L^{\infty}\right)_{H}, H$ is optimal. 
Example 17. Consider the space $H=L_{*}^{1}(v)$, where $\rho_{H}(g)=$ $\int_{0}^{\infty} v(t) g(t) d t / t$ and $\rho_{H} \in N_{t}$. Using Theorem 15, we can construct an optimal domain $E$, where

$$
\begin{aligned}
\rho_{E}(g) & =\rho_{H}\left(R_{\min (k, n)} g\right) \\
& =\int_{0}^{\infty} t^{\min (k, n) / n} w(t) g(t) \frac{d t}{t}
\end{aligned}
$$

and $w(t)=\int_{t}^{\infty} v(u) d u / u$. Hence $E=\Lambda^{1}\left(t^{\min (k, n) / n} w\right)$, and this couple is optimal. Also $\alpha_{E}=\beta_{E}=\min (k, n) / n$ if $v$ is slowly varying. Note that if $k \geq n$, then $E=\Lambda^{1}(t w)=\Gamma^{1}(t v)=$ $\left(L^{1}, L^{\infty}\right)_{H}$

Example 18. Let $H=L^{\infty}(v)$, where $\rho_{H}(g)=\sup v(t) g(t)$ and $\rho_{H} \in N_{t}$ and let

$$
\rho_{E}(g)=\sup v(t) \int_{0}^{t} u^{\min (k, n) / n} g(u) \frac{d u}{u} .
$$

Then by Theorem 15, the domain $E$ is optimal and the couple is optimal. In particular, the couple $L^{n / \min (k, n), 1}, C^{0}$ is optimal. If $k=n+j, j \geq 0$, this means that the embedding $W_{1}^{n+j} \hookrightarrow C^{j}$ is optimal.

Example 19. Let $H$ be as in the previous example. Since

$$
\begin{gathered}
\rho_{E}(g) \leq \sup t^{\min (k, n) / n} w(t) g(t), \\
\frac{1}{v(t)}=\int_{0}^{t} \frac{1}{w(u)} \frac{d u}{u},
\end{gathered}
$$

it follows that the couple $E=\Lambda^{\infty}\left(t^{\min (k, n) / n} w\right), H=L_{*}^{\infty}(v)$ is admissible. In order to prove that $\rho_{H}$ is optimal, take any $g \in$ $M_{0}$, and define $h$ from $t^{\min (k, n) / n} w(t) h(t)=\sup _{u \geq t} v(u) g(u)$. Then $h \in D_{1}$ and $\rho_{E_{1}}(h) \lesssim \rho_{H}(g)$. On the other hand

$$
\begin{aligned}
R_{\min (k, n)} h(t) & =\int_{0}^{t} \sup _{x \geq u} v(x) g(x) \frac{1}{w(u)} \frac{d u}{u} \\
& \geq \sup _{u \geq t} v(u) g(u) \frac{1}{v(t)} \geq g(t) .
\end{aligned}
$$

Hence $\rho_{H(E)}(g) \leq \rho_{E_{1}}(h) \lesssim \rho_{H}(g)$; therefore $\rho_{H}$ is optimal.

Example 20 (case $k \geq n$ ). Let $H=L_{*}^{q}(v), 0<q \leq \infty$, where $\rho_{H}(g)=\left(\int_{0}^{\infty}[v(t) g(t)]^{q} d t / t\right)^{1 / q}$ and $\rho_{H} \in N_{t}$ and let $k \geq n$. Using Remark 16, we can construct an optimal domain $E=$ $\Gamma^{q}(t v)$ and this couple is optimal. Also $\alpha_{E}=\beta_{E}=1$ if $v$ is slowly varying.

\subsection{Optimal Target Quasinorms}

Definition 21 (construction of the optimal target quasi-norm). For a given domain quasinorm $\rho_{E} \in N_{d}$, we set

$$
\begin{array}{r}
\rho_{H(E)}(g):=\inf \left\{\rho_{E}(h): g \leq R_{\min (k, n)} h, h \in D_{1}\right\}, \\
g \in M_{\min (k, n) .}
\end{array}
$$

Note that $\alpha_{H(E)}=\beta_{H(E)}=\min (k, n) / n-\alpha_{E}$.
Theorem 22. The target quasinorm $\rho_{H(E)}$ belongs to $N_{t}$, the couple $\rho_{E}, \rho_{H(E)}$ is admissible, and the target quasinorm is optimal.

Proof. The property " $\rho_{H(E)}(g)=0$ implies $g=0$ " follows from (30). Also, since $\rho_{E} \in N_{d}$ it is easy to check that $\rho_{H(E)} \in N_{t}$. The couple is admissible since $\rho_{H(E)}\left(R_{\min (k, n)} h\right) \leq$ $\rho_{E}(h), h \in D_{1}$. Suppose that the couple $\rho_{E}, \rho_{H_{1}} \in N_{t}$ is admissible. Then $\rho_{H_{1}}\left(\chi_{(0,1)} R_{\min (k, n)} h\right) \lesssim \rho_{E}(h), h \in D_{1}$. Therefore if $\chi_{(0,1)} g \leq R_{\min (k, n)} h, h \in D_{1}$, then $\rho_{H_{1}}\left(\chi_{(0,1)} g\right) \leq$ $\rho_{H_{1}}\left(\chi_{(0,1)} R_{\min (k, n)} h\right) \lesssim \rho_{E}(h)$, whence $\rho_{H_{1}}\left(\chi_{(0,1)} g\right) \lesssim \rho_{H(E)}(g)$, $g \in M_{\min (k, n)}$. Hence $\rho_{H(E)}$ is optimal.

Theorem 23 (supercritical case). If $\alpha_{E}<\min (k, n) / n$ and $\rho_{E}\left(\chi_{(1, \infty)}(t) t^{-\min (k, n) / n}\right)<\infty$, then

$$
\rho_{H(E)}(g) \approx \rho_{E}\left(t^{-\min (k, n) / n} g(t)\right), \quad g \in M_{\min (k, n)} .
$$

Moreover, the couple $\rho_{E}, \rho_{H(E)}$ is optimal.

Proof. If $g \leq R_{\min (k, n)} h, h \in D_{1}$, then, by Minkowski's inequality and $\operatorname{since} \min (k, n) / n>\alpha_{E}$, it follows

$$
\begin{aligned}
& \rho_{E}\left(t^{-\min (k, n) / n} g(t)\right) \\
& \quad \leq \rho_{E}\left(t^{-\min (k, n) / n} R_{\min (k, n)} h(t)\right) \lesssim \rho_{E}(h) .
\end{aligned}
$$

Hence, taking the infimum, we get $\rho_{E}\left(t^{-\min (k, n) / n} g(t)\right) \lesssim$ $\rho_{H(E)}(g)$.

On the other hand, for $g \in M_{\min (k, n)}$, we have $g \lesssim$ $R_{\min (k, n)} h, h(t)=t^{-\min (k, n) / n} g(t)$. Since $h \in D_{1}$ it follows $\rho_{H(E)}(g) \lesssim \rho_{E}(h) \lesssim \rho_{E}\left(t^{-\min k, n / n} g(t)\right)$.

The domain quasinorm $\rho_{E}$ is also optimal since for $f \in$ $L^{1}(\Omega)$,

$$
\begin{aligned}
\rho_{E(H(E))}\left(f^{*}\right) & =\rho_{H(E)}\left(R_{\min (k, n)} f^{*}\right) \\
& \approx \rho_{E}\left(t^{-\min (k, n) / n} R_{\min (k, n)} f^{*}(t)\right) \gtrsim \rho_{E}\left(f^{*}\right) .
\end{aligned}
$$

Example 24. Consider the space $E=\Lambda^{q}(w), 0<q \leq \infty$, $\min (k, n) / n>\beta_{E}=\alpha_{E}>0$. Then by Theorem 23 the couple $E, H, H=L_{*}^{q}\left(t^{-\min (k, n) / n} w\right)$ is optimal. In particular, using also Theorem 13, the embedding $W^{k} L^{p, \infty} \hookrightarrow \mathscr{C}^{k-n / p}, k>$ $n / p, 1<p<\infty$, is optimal.

In the critical case we do not know how to simplify the optimal target quasi-norm, defined in (74). Instead, we can construct a large class of domain quasinorms and the corresponding optimal target quasinorms by using extrapolation from the super-critical case. Recall some basic definitions and results from the extrapolation theory [3]. Let $\left(A_{0}, A_{1}\right)$ be a couple of quasi-Banach spaces. The sigma extrapolation space $\Sigma^{q}\left(M(\sigma)\left(A_{0}, A_{1}\right)_{a(t) t^{-\sigma}, q}\right), a$-positive weight, $0<\sigma<\sigma_{0}$, $0<q \leq \infty, M$-positive decreasing weight, consists of all 
$f \in A_{0}+A_{1}$ such that $f=\sum_{j=l}^{\infty} g_{j}, g_{j} \in A_{j}, A_{j}:=\left(A_{0}\right.$, $\left.A_{1}\right)_{a(t) t^{-1 / 2 j}, q^{j}}$ with a quasinorm

$$
\|f\|_{\Sigma^{q}\left(M(\sigma)\left(A_{0}, A_{1}\right)_{a(t) t^{-\sigma}, q}\right)}=\inf \left(\sum_{j=l}^{\infty}\left[M\left(2^{-j}\right)\left\|g_{j}\right\|_{A_{j}}\right]^{q}\right)^{1 / q},
$$

where the infimum is taken with respect to all representations $f=\sum_{j=l}^{\infty} g_{j}$.

This space can be characterized as an interpolation space.

Theorem 25 (see [3]). Let $a(t)=t^{-\theta} b(t), b$-slowly varying, $0<\theta<1$. Then

$$
\Sigma^{q}\left(M(\sigma)\left(A_{0}, A_{1}\right)_{a(t) t^{-\sigma}, q}\right)=\left(A_{0}, A_{1}\right)_{w, q},
$$

where

$$
\frac{1}{w(t)}=\frac{1}{a(t)}\left(\int_{0}^{\sigma_{0}}\left[\frac{t^{\sigma}}{M(\sigma)}\right]^{r} \frac{d \sigma}{\sigma}\right)^{1 / r}
$$

and $1 / r+1 / q=1$ if $q>1, r=\infty$ if $0<q \leq 1$.

Our main result is the following one.

Theorem 26. Let $E=\Lambda^{q}\left(t^{k / n} c(t)(1-\ln t)\right), k<n, c$-slowly varying weight, $c(+0)=\infty, c\left(t^{2}\right) \approx c(t), 0<q \leq \infty, H=$ $L_{*}^{q}(c)$. We suppose that $\rho_{E} \in N_{d}$ and $\rho_{H} \in N_{t}$. Then this couple is admissible and the target quasinorm is optimal.

Proof. Step 1 (admissibility). Since $\alpha_{E}=\beta_{E}=k / n<1$, it will be enough to check that

$$
\rho_{H}\left(R_{k}\left(g^{* *}\right)\right) \lesssim \rho_{E}\left(g^{* *}\right),
$$

where

$$
\begin{gathered}
\rho_{E}(g)=\left(\int_{0}^{1}\left[t^{k / n} c(t)(1-\ln t) g(t)\right]^{q} \frac{d t}{t}\right)^{1 / q}, \\
\rho_{H}(g)=\left(\int_{0}^{1}[c(t) g(t)]^{q} \frac{d t}{t}\right)^{1 / q} .
\end{gathered}
$$

Applying Minkowski's inequality we obtain for $0<\sigma<$ $\sigma_{0}<k / n, b$-slowly varying weight,

$$
\sigma\left\|R_{k}\left(g^{* *}\right)\right\|_{L_{*}^{q}\left(b(t) t^{-\sigma}\right)} \leqslant\|g\|_{\Gamma^{q}\left(t^{k / n-\sigma} b(t)\right)} .
$$

In order to extrapolate these inequalities, we write

$$
\begin{gathered}
\Gamma^{q}\left(t^{k / n-\sigma} b(t)\right)=\left(L^{1}, L^{\infty}\right)_{b(t) t^{k / n-1-\sigma}, q^{\prime}} \\
L_{*}^{q}\left(t^{-\sigma} b(t)\right)=\left(L_{*}^{q}\left(t^{1 / 2} b(t)\right), L_{*}^{q}\left(t^{-1 / 2} b(t)\right)\right)_{1 / 2+\sigma, q} \\
\sigma_{0}<\frac{1}{2} .
\end{gathered}
$$

This is true since

$$
\begin{aligned}
& K\left(t, g ; L_{*}^{q}\left(w_{0}\right), L_{*}^{q}\left(w_{1}\right)\right) \\
& \quad \approx\left(\int_{0}^{1}\left[g(u) \min \left(w_{0}(u), t w_{1}(u)\right)\right]^{q} \frac{d u}{u}\right)^{1 / q},
\end{aligned}
$$

$0<t<1$.

Let $\sigma=2^{-j}$ and $g=\sum g_{j}$ (convergence in $L^{1}$ ), where $g_{j} \in L^{\infty}$. Then $g^{* *} \leq \sum g_{j}^{* *}$, whence $R_{k}\left(g^{* *}\right) \leq \sum R_{k}\left(g_{j}^{* *}\right)$ and for $M(\sigma)=\sigma^{-2}, p=\min (q, 1)$, we have

$$
K^{p}\left(t, R_{k}\left(g^{* *}\right) ; B_{0}, B_{1}\right) \leq C_{v}:=\sum_{j \geq l} K^{p}\left(t, R_{k}\left(g_{j}^{* *}\right) ; B_{0}, B_{1}\right),
$$

where $B_{0}=L_{*}^{q}\left(t^{1 / 2} b(t)\right), B_{1}=L_{*}^{q}\left(t^{-1 / 2} b(t)\right)$. We can write

$$
\begin{aligned}
C_{\nu}=\sum_{j \geq l} & {\left[t^{-1 / 2-2^{-j}} 2^{-j} M\left(2^{-j}\right) K\left(t, R_{k}\left(g_{j}^{* *}\right) ; B_{0}, B_{1}\right)\right]^{p} } \\
& \times\left[\frac{t^{1 / 2+2^{-j}}}{2^{-j} M\left(2^{-j}\right)}\right]^{p}
\end{aligned}
$$

and using also Hölder's inequality if $q>1$, we get

$$
\begin{aligned}
& {[v(t)]^{p} C_{v}} \\
& \quad \leq \sum_{j \geq l}\left[t^{-1 / 2-2^{-j}} 2^{-j} M\left(2^{-j}\right) K\left(t, R_{k}\left(g_{j}^{* *}\right) ; B_{0}, B_{1}\right)\right]^{p},
\end{aligned}
$$

where

$$
\frac{1}{v(t)}=\left(\sum_{j \geq l}\left[\frac{t^{1 / 2+2^{-j}}}{2^{-j} M\left(2^{-j}\right)}\right]^{r}\right)^{1 / r} .
$$

Hence

$$
\begin{aligned}
& \left\|R_{k}\left(g^{* *}\right)\right\|_{\left(B_{0}, B_{1}\right)_{v, q}} \\
& \quad \leq\left(\sum_{j \geq l}\left[2^{-j} M\left(2^{-j}\right)\left\|R_{k}\left(g_{j}^{* *}\right)\right\|_{\left(B_{0}, B_{1}\right)_{1 / 2+2^{-j}, q}}\right]^{q}\right)^{1 / q} .
\end{aligned}
$$

Since

$$
2^{-j}\left\|R_{k}\left(g_{j}^{* *}\right)\right\|_{\left(B_{0}, B_{1}\right)_{1 / 2+2^{-j}, q}} \leq\left\|g_{j}\right\|_{\Gamma^{q}\left(t^{k / n-2^{-j}} b(t)\right)},
$$

we get

$$
\left\|R_{k}\left(g^{* *}\right)\right\|_{\left(B_{0}, B_{1}\right)_{v, q}} \leq\|g\|_{\Sigma^{q}\left(M(\sigma)\left(L^{1}, L^{\infty}\right)_{b(t) t^{k / n-1-\sigma_{, q}}}\right)},
$$

whence

$$
R_{k}:\left(L^{1}, L^{\infty}\right)_{w, q} \longmapsto\left(L_{*}^{q}\left(t^{1 / 2} b(t)\right), L_{*}^{q}\left(t^{-1 / 2} b(t)\right)\right)_{v, q^{\prime}}
$$


where $w$ is given by (80) with $a(t)=b(t) t^{k / n-1}$ and $M(\sigma)=$ $\sigma^{-2}$. It is easy to calculate these weights, see [3]. We have

$$
\begin{array}{r}
w(t) \approx b(t) t^{k / n-1}(1-\ln t)^{2}, \quad v(t) \approx t^{-1 / 2} \\
(1-\ln t), \\
0<t<1 .
\end{array}
$$

Then for $b(t)=c(t)(1-\ln t)^{-1}$ we get

$$
\begin{gathered}
\Gamma^{q}\left(t^{k / n} c(t)(1-\ln t)\right) \hookrightarrow\left(L^{1}, L^{\infty}\right)_{w, q}, \\
\left(L_{*}^{q}\left(t^{1 / 2} b(t)\right), L_{*}^{q}\left(t^{-1 / 2} b(t)\right)\right)_{v, q} \hookrightarrow L_{*}^{q}(c) .
\end{gathered}
$$

Hence (81) is proved.

Step 2 (optimality of the target quasi-norm). We want to prove that $\rho_{H}$ is an optimal target quasi-norm. It is sufficient to see that

$$
\rho_{H(E)}\left(\chi_{(0,1)} g\right) \lesssim \rho_{H}(g), \quad g \in M_{k} \text {, }
$$

where $\rho_{H(E)}$ is defined by (74). To this end for any such $g$ we construct an $h \in D_{1}$ such that $\chi_{(0,1)} g \lesssim R_{k} h$ and $\rho_{E}(h) \lesssim \rho_{H}(g)$. Let $0<q<\infty$. (The case $q=\infty$ is analogous, see Example 19.) Let $h^{q}(t)=\chi_{(0,2)}(t) \int_{t}^{2}(1-$ $\ln u)^{-q} u^{-q} g^{q}(\sqrt{u e}) d u / u$. Then $h \in D_{1}$ and $\rho_{E}(h) \lesssim \rho_{H}(g)$. On the other hand, for $0<t<1$,

$$
R_{k} h(t) \geq \int_{t^{2} / e}^{t} \frac{g(\sqrt{e u})}{1-\ln u} \frac{d u}{u} \gtrsim \chi_{(0,1)}(t) g(t),
$$

since $\int_{t^{2} / e}^{t}(1-\ln u)^{-1} d u / u=\ln 2$. Then by the definition of $\rho_{H(E)}$ we get

$$
\rho_{H(E)}\left(\chi_{(0,1)} g\right) \lesssim \rho_{E}(h) \lesssim \rho_{H}(g) .
$$

In the limiting case $k=n$ we can use the fact that the weights $b(t)=c(t)(1-\ln t)$ and $c(t)$, where $c$ is decreasing and slowly varying, are Muckenhoupt's weights; that is,

$$
\begin{aligned}
\left(\int_{t}^{1} c^{q}(u) \frac{d u}{u}\right)^{1 / q}\left(\int_{0}^{t}[b(u)]^{-r} \frac{d u}{u}\right)^{1 / r} \leqslant 1, & \frac{1}{q}+\frac{1}{r}=1 \\
0 & <t<1 .
\end{aligned}
$$

Then the operator $R_{n}$ is bounded from $L_{*}^{q}(b)$ to $L_{*}^{q}(c), 1 \leq$ $q \leq \infty$ (see [28]). In this way we have the following result, with optimality being proved as in the case $k<n$.

Theorem 27. Let $E=\Lambda^{q}(t c(t)(1-\ln t))$, c-decreasing slowly varying weight, $c(+0)=\infty, c\left(t^{2}\right) \approx c(t), 1 \leq q \leq \infty, H=$ $L_{*}^{q}(c)$. We suppose that $\rho_{E} \in N_{d}$ and $\rho_{H} \in N_{t}$. Then this couple is admissible and the target quasinorm is optimal.

\section{Authors' Contribution}

G. E. Karadzhov research is partially supported by the Abdus Salam School of Mathematical Sciences, GC University Lahore, by a grant from HEC, Pakistan.

\section{Acknowledgment}

The authors are grateful to the reviewers for the useful remarks that improved the paper.

\section{References}

[1] H. Triebel, Theory of Function Spaces, vol. 78, Birkhäuser, Basel, Switzerland, 1983.

[2] E. M. Stein, "Editor's note: the differentiability of functions in $\mathbf{R}^{n}$, , Annals of Mathematics, vol. 113, no. 2, pp. 383-385, 1981.

[3] G. E. Karadzhov and M. Milman, "Extrapolation theory: new results and applications," Journal of Approximation Theory, vol. 133, no. 1, pp. 38-99, 2005.

[4] K. Hansson, "Imbedding theorems of Sobolev type in potential theory," Mathematica Scandinavica, vol. 45, no. 1, pp. 77-102, 1979.

[5] A. Cianchi, "Symmetrization and second-order Sobolev inequalities," Annali di Matematica Pura ed Applicata, vol. 183, no. 1, pp. 45-77, 2004.

[6] C. Capone, A. Fiorenza, G. E. Karadzhov, and W. Nazeer, "Optimal couples of rearrangement invariant spaces for the Riesz potential," Zeitschrift für Analysis und ihre Anwendungen, vol. 30, no. 2, pp. 219-236, 2011.

[7] J. Vybiral, Optimality of Function Spaces for Boundedness of Integral Operators and Sobolev Embeddings, Faculty of Mathematics and Physics, Charles University, Prague, Czech Republic, 2002.

[8] Y. V. Netrusov, "Embedding theorems for Besov spaces in symmetric spaces," Zapiski Nauchnykh Seminarov LOMI, vol. 159, pp. 69-102, 1987.

[9] V. I. Kolyada, "Rearrangements of functions, and embedding theorems," Russian Mathematical Surveys, vol. 44, pp. 73-111, 1989.

[10] M. Cwikel and E. Pustylnik, "Sobolev type embeddings in the limiting case," The Journal of Fourier Analysis and Applications, vol. 4, no. 4-5, pp. 433-446, 1998.

[11] D. E. Edmunds, R. Kerman, and L. Pick, "Optimal Sobolev imbeddings involving rearrangement-invariant quasinorms," Journal of Functional Analysis, vol. 170, no. 2, pp. 307-355, 2000.

[12] M. L. Goldman and R. A. Kerman, "On the optimal embedding of Calderón spaces and of generalized Besov spaces," Proceedings of the Steklov Institute of Mathematics, vol. 243, pp. 154-184, 2003.

[13] M. Milman and E. Pustylnik, "On sharp higher order Sobolev embeddings," Communications in Contemporary Mathematics, vol. 6, no. 3, pp. 495-511, 2004.

[14] J. Martín and M. Milman, "Symmetrization inequalities and Sobolev embeddings," Proceedings of the American Mathematical Society, vol. 134, no. 8, pp. 2335-2347, 2006.

[15] R. Kerman and L. Pick, "Optimal Sobolev imbeddings," Forum Mathematicum, vol. 18, no. 4, pp. 535-579, 2006.

[16] J. Martin, M. Milman, and E. Pustylnik, "Self improving Sobolev inequalities, truncation and symmetrization," Journal of Functional Analysis, vol. 252, no. 2, pp. 677-695, 2007.

[17] J. Martín and M. Milman, "Higher-order symmetrization inequalities and applications," Journal of Mathematical Analysis and Applications, vol. 330, no. 1, pp. 91-113, 2007.

[18] A. Gogatishvili and V. I. Ovchinnikov, "Interpolation orbits and optimal Sobolev's embeddings," Journal of Functional Analysis, vol. 253, no. 1, pp. 1-17, 2007. 
[19] J. Martin and M. Milman, "Self improving Sobolev-Poincaré inequalities, truncation and symmetrization," Potential Analysis, vol. 29, no. 4, pp. 391-408, 2008.

[20] I. Ahmed and G. E. Karadzhov, "Optimal embeddings of generalized homogeneous Sobolev spaces," Colloquium Mathematicum, vol. 123, no. 1, pp. 1-20, 2011.

[21] Ju. Brudnyi and N. Ya. Krugliak, Interpolation Spaces and Interpolation Functors, North-Holland, Amsterdam, Netherlands, 1991.

[22] A. Gogatishvili, J. S. Neves, and B. Opic, "Optimal embeddings of Bessel-potential-type spaces into generalized Hölder spaces involving $k$-modulus of smoothness," Potential Analysis, vol. 32, no. 3, pp. 201-228, 2010.

[23] Z. Bashir, F. Cobos, and G. E. Karadzhov, "Optimal embeddings of Calderónspaces into the generalized Hölder-Zygmund spaces," Mathematica Scandinavica. In press.

[24] G. Köthe, Topologische Lineare Räume, Springer, Berlin, Germany, 1966.

[25] C. Bennett and R. Sharpley, Interpolation of Operators, vol. 129, Academic Press, New York, NY, USA, 1988.

[26] R. A. DeVore and R. C. Sharpley, "On the differentiability of functions in $\mathbf{R}^{n}$," Proceedings of the American Mathematical Society, vol. 91, no. 2, pp. 326-328, 1984.

[27] J. Martin and M. Milman, "Fractional sobolev inequalities: symmetrization,isoperimetry and interpolation," http://arxiv.org/ $\mathrm{abs} / 1205.1231$.

[28] B. Muckenhoupt, "Hardy's inequality with weights," Studia Mathematica, vol. 44, pp. 31-38, 1972. 


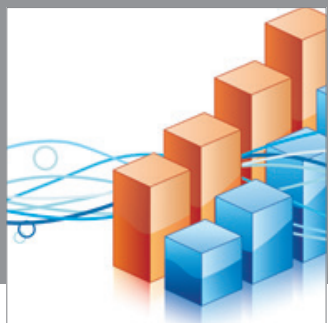

Advances in

Operations Research

mansans

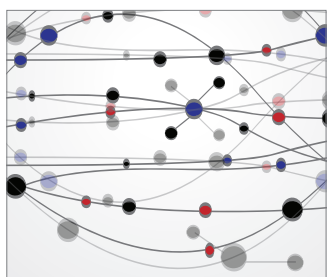

The Scientific World Journal
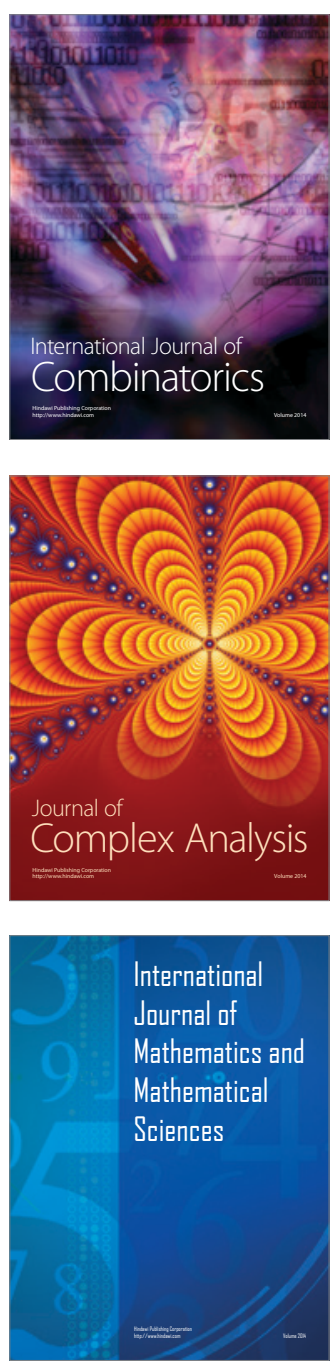
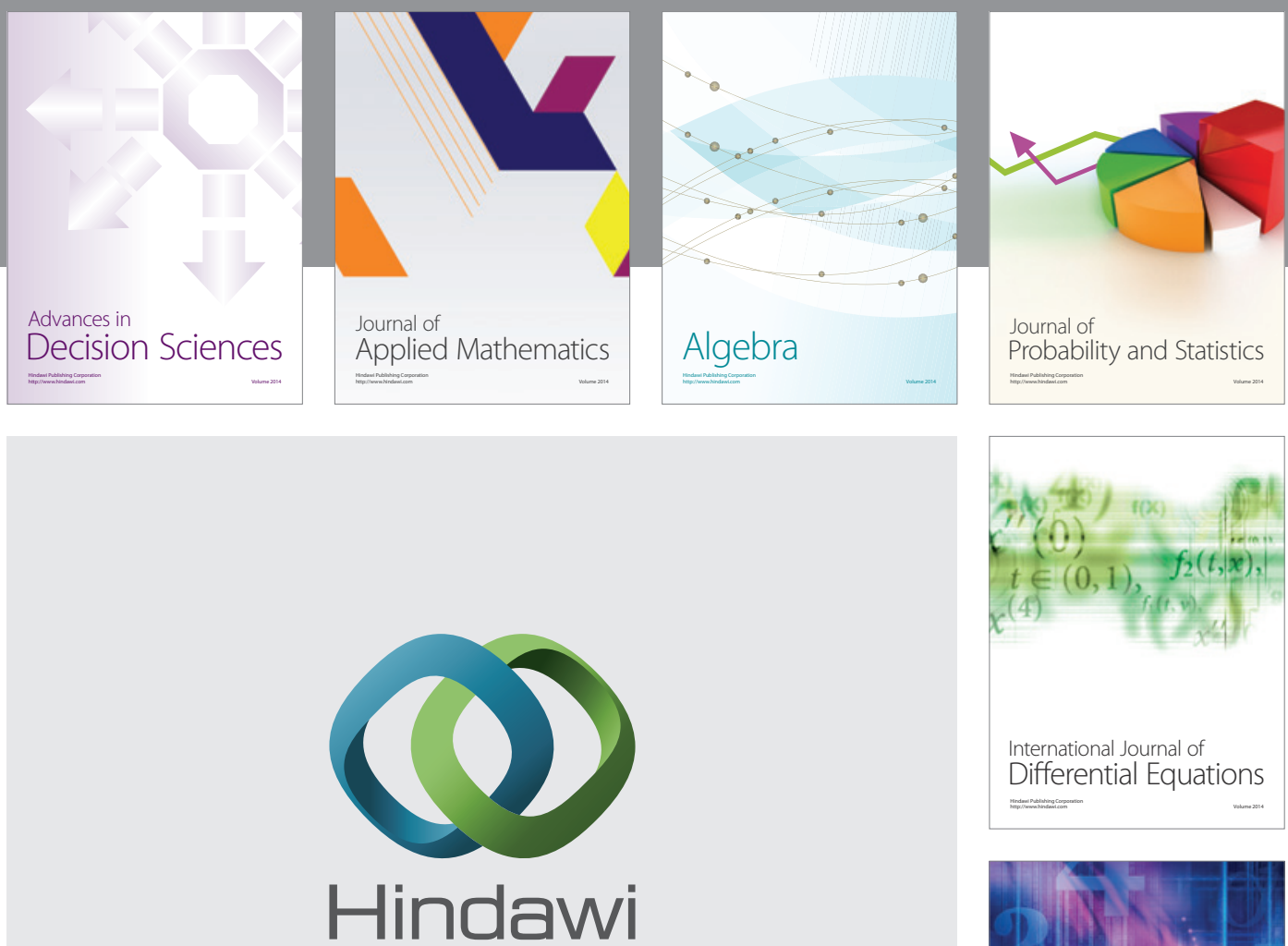

Submit your manuscripts at http://www.hindawi.com
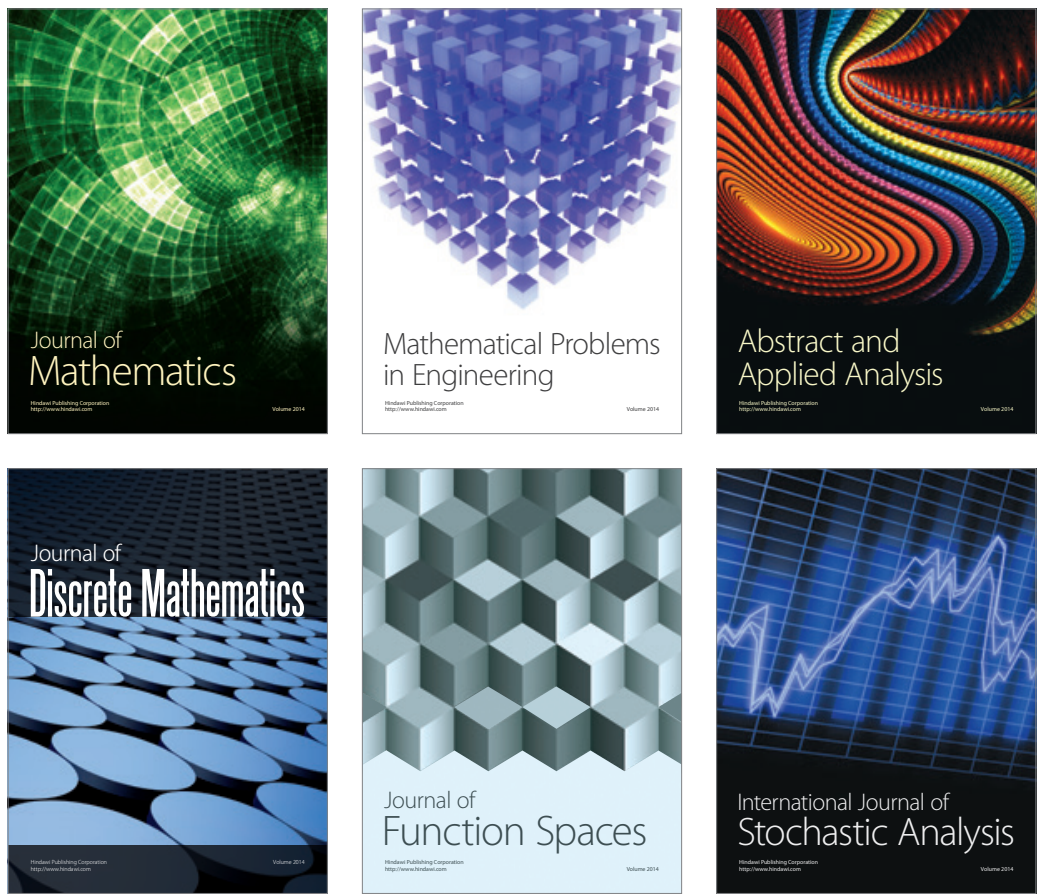

Journal of

Function Spaces

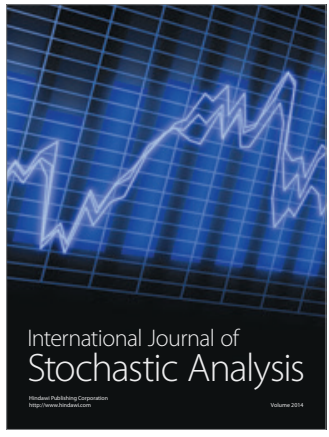

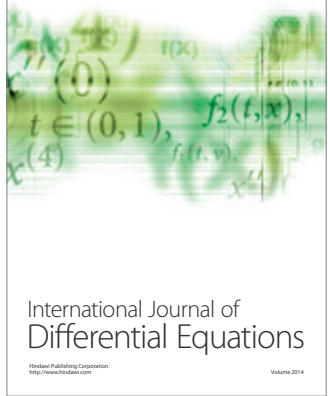
